\title{
Magnetic Resonance Imaging in Acute and Chronic Kidney Diseases: Present Status
}

\author{
Jean-Pierre Laissy ${ }^{a}$ Jean-Marc Idée ${ }^{c}$ Pedro Fernandez ${ }^{a}$ Muriel Floquet $^{a}$ \\ François Vrtovsnik $^{b}$ Elisabeth Schouman-Claeys ${ }^{a}$ \\ Departments of ${ }^{a}$ Radiology and ${ }^{b}$ Nephrology, Hôpital Bichat, Paris, and ${ }^{c}$ Guerbet, Roissy, France
}

\section{Key Words}

Kidney, diseases $\cdot$ Kidney, functional studies $\cdot M R$ imaging $\cdot$ Glomerular filtration $\cdot$ Contrast media, MR •

Diffusion-weighted imaging

\begin{abstract}
Magnetic resonance imaging (MRI) of normal and diseased kidneys shows great promise because of the combined value of anatomical and functional information provided, as well as of specific contrast patterns that can be observed non-invasively. Multicontrast MRI is able to show infiltrative kidney disorders. Diffusion-weighted imaging can assess alterations in renal function and can suggest obstruction or inflammation when present. Due to the low nephrotoxicity, contrast-enhanced MR studies using serial dynamic enhancement with non-specific gadolinium chelates are able to provide information on glomerular filtration. Furthermore, contrast agents such as ultrasmall particles of iron oxide, specific of inflammation, should be used in the near future to detect active from quiescent involvement, both in native kidneys and renal allografts. Early results should indicate that these compounds might differentiate acute tubular necrosis from other acute nephropathies, as well as active proliferative nephropathies from chronic ones. Ongoing stud-
\end{abstract}

ies will obviously demonstrate the value of the combination of these various MRI sequences in the diagnosis of acute renal failure and chronic kidney disease.

Copyright (C) 2006 S. Karger AG, Basel

Magnetic resonance imaging (MRI) is a diagnostic modality with a growing role in the assessment of both acute renal failure (ARF) and chronic kidney disease. It has long been restricted by its low availability, long acquisition times, and relatively low spatial acquisition. However, recent major technical developments using this technique have allowed ultrashort acquisition times and numerous studies have been carried out to validate the use of non-specific gadolinium chelates for the functional imaging of the kidneys [1]. The absence of nephrotoxicity of gadolinium chelate at the current dosages injected makes the technique particularly well suited in patients with acute or chronic kidney disease. Both anatomical and functional assessment are available with this technique.

There are several MR techniques which are useful for the diagnosis of acute and chronic kidney diseases. The most important ones are plain multicontrast MRI (including T1-, T2- and diffusion-weighted imaging), and contrast-enhanced MRI. Contrast agents used are either 

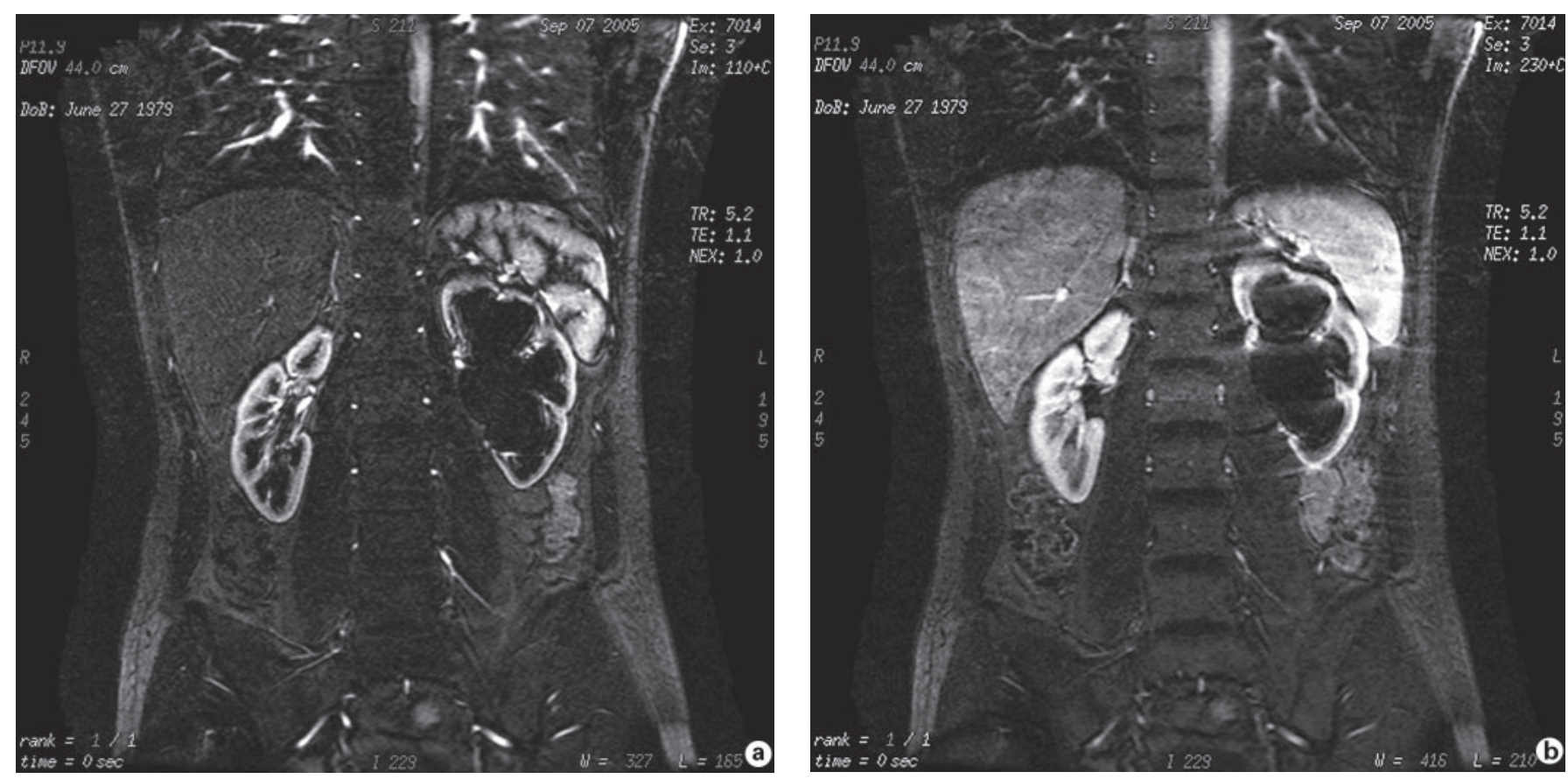

Fig. 1. Enlarged left kidney due to recent obstruction. Coronal T1-weighted imaging after gadolinium chelate injection. Thinning of the cortex with a symmetrical enhancement with the normal right kidney at the arterial (a) and tubular (b) phase.

non-specific gadolinium chelates, or ultrasmall superparamagnetic particles of iron oxide (USPIO) that may reflect active tissue inflammation. The latter are not yet fully available in clinical practice. Other currently used techniques such as contrast-enhanced MR angiography on the one hand and BOLD imaging on the other hand are discussed in another section of this journal.

\section{Plain and Conventional Gadolinium-Enhanced MRI}

MRI, as well as other non-invasive modalities such as ultrasound and CT, can provide morphological information on kidneys. The size of the kidneys can be an indicator of the underlying disease. Small kidneys are generally the result of renal artery or glomerular disease, irregularshaped kidneys are seen in interstitial kidney disease, and enlarged kidneys are due to either obstruction (fig. 1), diabetes mellitus, infection, amyloidosis, or renal vein thrombosis. The characterization of masses and cysts, such as in polycystic kidney disease, is usually easy (fig. 2). Several renal diseases may cause spontaneous abnormal signal intensity on MR images, the appearance of

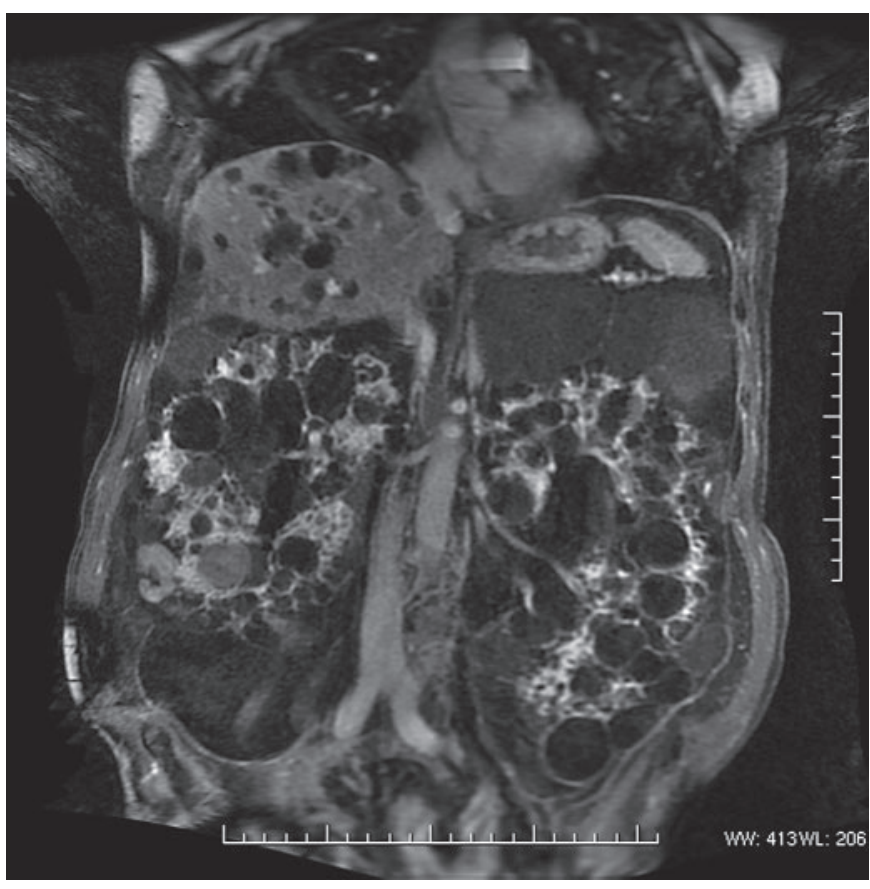

Fig. 2. Patient with polycystic kidney disease. Coronal contrastenhanced T1-weighted image with fat saturation shows enhancement of the residual functioning renal parenchyma between the cysts, as well as a stretched left renal artery. 
which being sufficiently characteristic to allow a specific radiologic diagnosis [2]. Low signal MR images are seen in three main categories: hemolysis (paroxysmal nocturnal hemoglobinuria, cortical hemosiderin deposition from mechanical hemolysis, and sickle cell disease), infection (hemorrhagic fever with renal syndrome), and vascular disease (renal arterial infarction, acute renal vein thrombosis, renal cortical necrosis, transplanted kidney rejection, and acute non-myoglobinuric renal failure). Conventional contrast-enhanced T1-weighted imaging adds little value to the technique. A recent study showed that the loss of corticomedullary differentiation (CMD) was independent of serum creatinine level on patients with ARF [3]. Therefore, there is need for other, more specific MR techniques.

\section{Diffusion-Weighted MRI}

The diagnosis of various renal diseases such as chronic renal failure, renal artery stenosis, and ureteral obstruction can benefit from measuring the diffusion characteristics of the kidney [4]. Diffusion-weighted (DW) MR imaging of the kidneys is able to provide information on renal function and can be suggestive of the presence and degree of obstruction or inflammation. The apparent diffusion coefficient (ADC), a quantitative parameter calculated from the DW MR images, combines the effects of capillary perfusion and water diffusion in the extracellular extravascular space. Thus, DW MR imaging provides information on perfusion and diffusion simultaneously [5]. Hydration is an important factor to increase global ADC values [6], whereas renal artery stenosis or ureteral obstruction decrease those values $[7,8]$. In case of acute or chronic renal failure, the cortical and medullary ADC values are significantly decreased when compared with normal kidneys and the cortical value decrease seems to be well correlated with serum creatinine levels [8]. In a rat study, the intravenous administration of the high-viscosity iodinated contrast agent iodixanol was found to significantly decrease the ADC, this effect occurring earlier in the cortex and lasting less than in the medulla [9].

\section{Dynamic Serial Gadolinium-Enhanced MRI}

MRI is able to non-invasively provide a functional assessment of the kidney, such as glomerular filtration rate (GFR), tubular concentration and transit, blood volume and perfusion, diffusion, and oxygenation. These ap- proaches can be achieved using endogenous contrast agents such as water protons or deoxyhemogobin or require exogenous contrast agents such as gadolinium $\left(\mathrm{Gd}^{+}\right)$chelates or superparamagnetic iron oxide nanoparticles [4]. Actually, $\mathrm{Gd}^{+}$chelates are good markers of the renal function in that they are freely filtered by the glomerulus and neither secreted nor reabsorbed by the nephron [10]. In a pig model of renal arterial stenosis, MRI associated with the intravenous administration of a $\mathrm{Gd}^{+}$chelate allowed the measurement of GFR as well as that of the extraction fraction. For this purpose, T1 of blood was measured in the infrarenal inferior vena cava and renal veins prior and after injection of the contrast agent. A significant linear correlation was found between extraction fraction measured with MRI vs. inulin [11]. Grenier et al. [12] studied 15 hypertensive patients with renal artery stenosis and found that captopril-sensitized dynamic MRI of the kidney with a $\mathrm{Gd}^{+}$chelate was feasible in patients with renovascular hypertension. There was a good correlation between MR and nuclear renography, although captopril-induced changes were not seen in all patients shown to have the disease.

Renal perfusion is one of the most common useful methods to detect renal impairment. It can be evaluated with contrast agents, based on tracer kinetic methods such as scintigraphy [13]. By injecting an MR contrast agent such as Gd-DOT/A or Gd-DTP/A, and following the change in image intensity over time, it is possible to obtain qualitative or semiquantitative information on the renal microcirculatory blood flow on a regional basis [10]. Fast acquisition techniques such as T1-weighted gradient echo or echo planar sequences allow sufficient temporal resolution to monitor intrarenal signal changes during the first pass of the agent through the kidneys (fig. 3). Gd3 ${ }^{+}$ chelates diffuse into the interstitial space (about 10\% during the first pass) and are freely filtered by the glomeruli (20\% during the first pass), as previously mentioned. However, the quantitative assessment of renal perfusion is impaired by the rapid diffusion of the contrast agent into the extracellular space and thus, only iron oxide particles or blood pool $\mathrm{Gd}^{+}$chelates (macromolecular or albumin-bound) can be considered as blood pool contrast agents and thus reliably applied to the measurement of renal perfusion [4]. Recently, P792, a macromolecular $\mathrm{Gd}^{+}$-based blood pool agent, has been shown to improve renal functional MRI assessment in rats by inducing less $\mathrm{T} 2 *$ effect without compromising T1 enhancement [14].

Currently-available non-specific contrast agents have been used to investigate renal perfusion in $\mathrm{ARF}$ and some studies have shown a loss of CMD that was suggested to 
Fig. 3. Magnification of a serial dynamic enhanced axial T1-weighted acquisition of a normal right kidney. Images obtained at the arterial (a), venous (b), tubular (c), and excretory (d) phase.
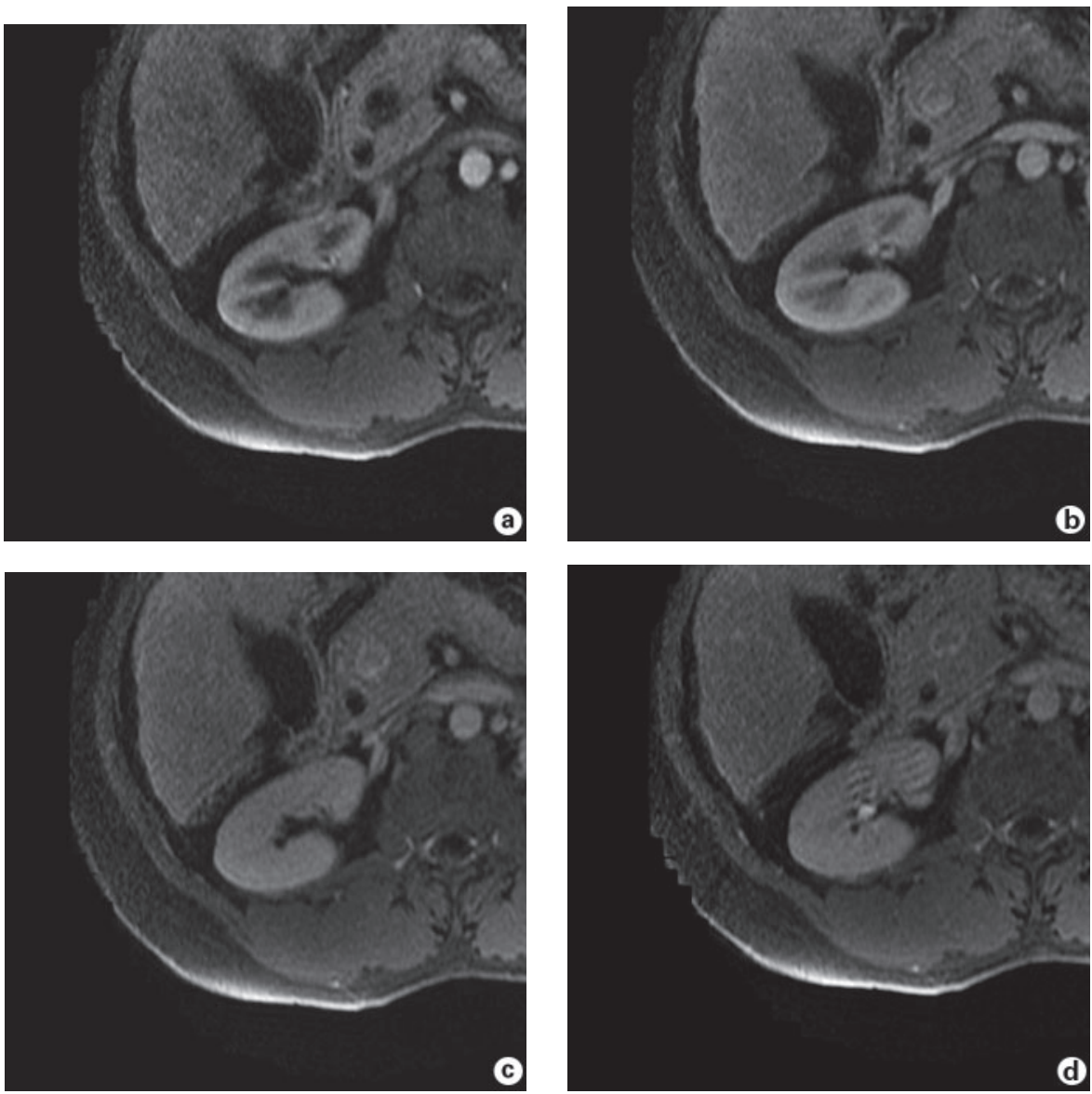

reflect changing differential perfusion patterns between the cortex and medulla in such pathological states [15]. However, a retrospective clinical study on 21 patients with ARF has shown that renal CMD can remain preserved on non-contrast T1-weighted or immediate $\mathrm{Gd}^{+}{ }^{+}$enhanced spoiled gradient echo images in patients with acute presentation of ARF, independent of serum creatinine level [3].

There is an obvious need for new, validated techniques for a better and more reliable evaluation of renal perfusion alterations during ARF. Blood pool contrast agents may demonstrate some usefulness in this setting.

\section{Gadolinium-Based Dendrimers}

These Gd compounds accumulate in the proximal tubules after early glomerular filtration. They act as positive contrast agents. Animal studies using 3D acquisitions with microMR devices showed various level concentra- tions between cortex, outer and inner medulla that disappear in case of acute as well as chronic renal injury. The main characteristics of dendrimer-enhanced MRI make it as a contrast medium specific of proximal tubule dysfunction $[1,16]$.

\section{Ultrasmall Superparamagnetic Particles of Iron Oxide}

USPIOs are iron oxides: magnetite $\left(\mathrm{Fe}_{3} \mathrm{O}_{4}\right)$, maghemite $\left(\gamma \mathrm{Fe}_{2} \mathrm{O}_{3}\right)$ or other ferrites that are insoluble in water. Unlike ferromagnetic substances and because of their size, superparamagnetic agents have no magnetic properties outside an external magnetic field. These nanoparticles have in common their specific uptake by macrophages, explaining, even if they are not entirely captured by liver and spleen, why they are widely evaluated as MRI markers for diagnosis of inflammatory and degenerative disorders associated with high macrophage phagocytic 
activity. At low concentration, they act as positive contrast agents (such as gadolinium), but at higher concentrations they result in a negative enhancement.

It is currently agreed that two main categories of superparamagnetic agents must be distinguished: (1) SPIOs (i.e. superparamagnetic iron oxides) the hydrodynamic size of which, including coating, is $>50 \mathrm{~nm}$, and (2) USPIOs (i.e. ultrasmall superparamagnetic iron oxides) which are $<50 \mathrm{~nm}$ in size.

This difference in size is reflected by significant differences in the ratio of relaxivity constants $\mathrm{r} 2 / \mathrm{r} 1$ but also by a significant difference in plasma half-life and biodistribution. Uptake of USPIOs such as ferumoxtran-10 (Sinerem ${ }^{\circledR}$ ) by the liver and the spleen is quite low because of their physicochemical characteristics (coating and hydrodynamic diameter). They consequently remain in the blood circulation for a relatively long time. Because of their long blood half-life and T1-shortening effect, these agents have been evaluated as blood pool agents for indications such as measurement of cerebral perfusion [17], myocardial or renal perfusion [18], angiography [19], or detection of vascular hepatic lesions [20].

These compounds are classically referred to as slowclearance blood pool agents. Whereas these agents are confined to the vascular compartment, their molecular volume only allows a slow 'sieving' through the glomerular fenestrae. Their renal clearance is consequently much lower than the normal GFR.

These blood pool characteristics provide a much longer time window for data acquisition during radiological procedures, with little loss of intravascular signal intensity. T2*-weighted images of normal kidneys show a maximum decrease in SI in the outer medulla, where the blood volume is the highest, because of susceptibility effects induced by the iron particles [4]. In a model of medullary ischemia induced by glycerol, the decrease in blood volume was clearly demonstrated within the outer medulla $[18,21]$. In radiocontrast nephrotoxicity, the degree of renal enhancement after iron oxide injection seems to be correlated with the reversibility of lesions $[22,23]$.

Intravenous injection of puromycin aminonucleoside in the rat is classically regarded as a good model of nephrotic syndrome secondary to glomerular epithelial cell lesions associated with glomerular and tubulointerstitial infiltration by macrophages [24]. In nephrotic rats, the USPIO ferumoxtran-10 injected intravenously $(90 \mu \mathrm{mol} /$ $\mathrm{kg}$ ) induced a significant decrease in signal intensity in all compartments of the kidney in the puromycin aminonucleoside group whereas the signal from the control group did not vary within the $24 \mathrm{~h}$ post-injection. This effect was correlated with the presence of macrophages containing iron oxide nanoparticles at histology [25]. Interestingly, the same USPIO was also found to decrease signal intensity in the cortex in a model of nephritic nephropathy (by injection of sheep anti-rat glomerular basement membrane serum). In this model, equivalent to Goodpasture's syndrome in humans, inflammatory lesions were exclusively located at the level of glomeruli. Conversely, in a rat model of obstructive nephropathy, which is known to induce diffuse interstitial lesions with macrophage infiltration in all kidney structures, the USPIO induced a decrease in signal intensity in all compartments [26].

Renal allograft rejection is associated with a massive inflammatory infiltration [27]. In a rat renal allograft model, USPIO, when injected on the fourth day following transplantation, induced a decrease in MR signal intensity at $24 \mathrm{~h}$ in some rejecting allografts whereas isografts and allografts with immunosuppressive treatment were not associated with signal reduction [28]. Promising results were obtained in a preliminary clinical study performed in 7 patients with suspected proliferative glomerulonephritis and in 5 patients with suspected renal graft rejection imaged $72 \mathrm{~h}$ following injection of ferumoxtran-10. From this clinical study, it was concluded that USPIO-enhanced MRI may allow acute tubular necrosis to be distinguished from other acute nephropathies in native and transplanted kidneys and may also allow active proliferative nephropathies to be differentiated from chronic ones [29]. Of course, such preliminary clinical data obtained in a small number of patients need to be confirmed by other studies.

Dextran-coated USPIOs serving as blood pool agent were also used to evaluate renal perfusion in dynamic MRI in normal and transplanted rat kidneys. A good agreement between renal graft perfusion and histopathological changes associated with graft rejection suggests that USPIO-enhanced dynamic MRI may be used to eval-

Fig. 4. Plain and USPIO-enhanced imaging of a normal rat kidney (a) and of an ARF rat kidney (b). Images are obtained from the left to right with plain T2-weighted images and increasing concentrations of USPIO. The outer and inner medulla are bright on plain T2-weighted MR images with intense signal drop on corresponding USPIO-enhanced T2-weighted MR images (a). The same images obtained in a rat with radiocontrast-induced nephropathy show a lack of decreased enhancement (b). 

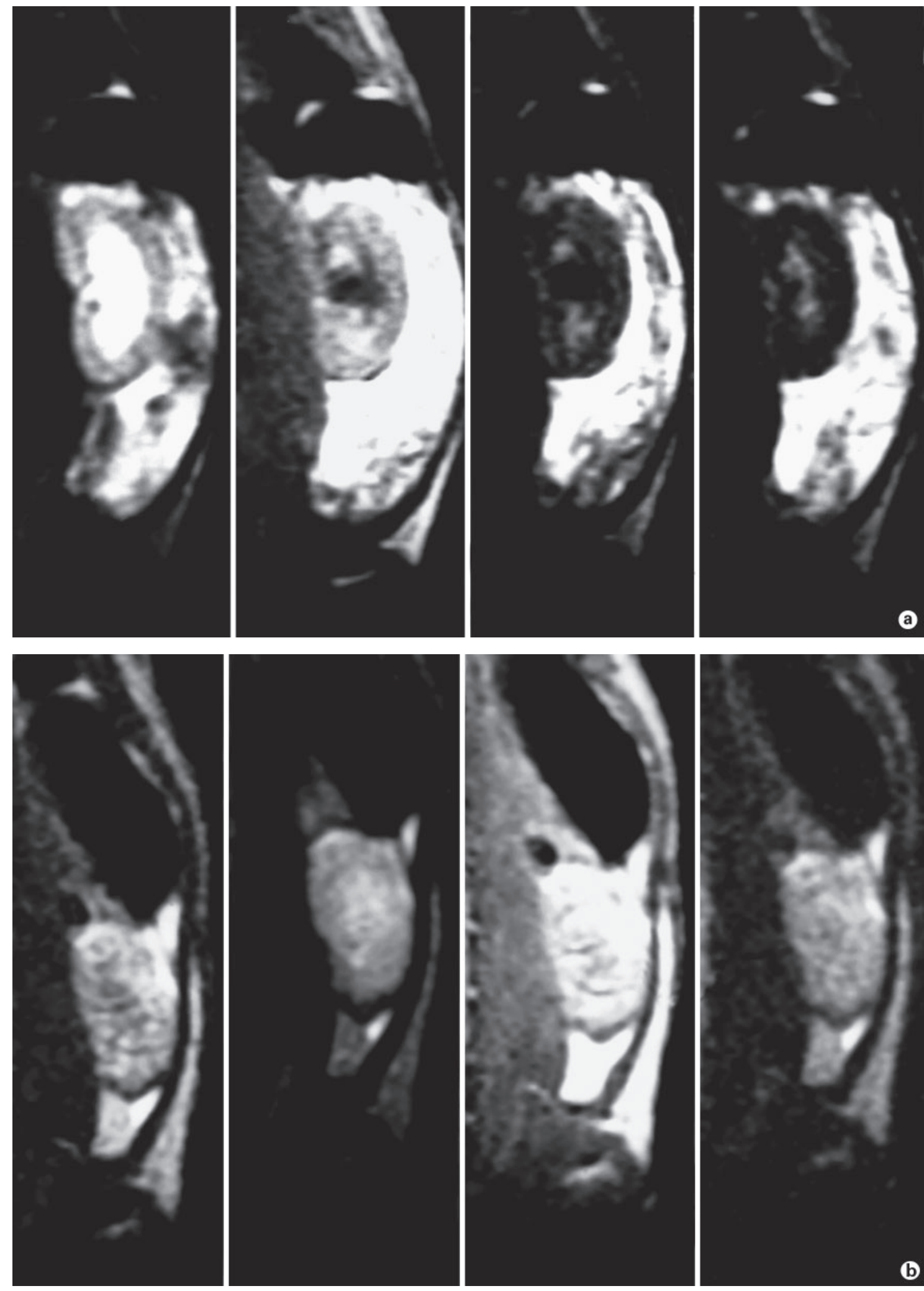
uate acute allograft rejection [30]. Iodinated contrast agents can be nephrotoxic in at-risk patients by inducing medullary hypoxia [31]. Intravenous injection of USPIO has been used in a validated rat model of contrast-induced nephropathy to assess renal lesions induced by the iodinated contrast agent as well as their reversibility (fig. 4). Interestingly, the degree of renal enhancement after iron oxide injection seemed to be correlated with the reversibility of lesions [22, 23].

At the present time, the vast majority of studies reported in the literature on USPIOs have been done on animals and few data are available on its real usefulness in clinical practice.

\section{Potential Clinical Applications}

The main issues of MRI in the near future will be to provide an accurate diagnosis of the underlying causes of acute or chronic renal failure, thus avoiding renal biopsy. Compared to this invasive procedure that harvests only samples within a single area of the kidney, MRI can provide repeatedly a complete view of both kidneys and monitor non-invasively progression or regression of diseases.

MRI already allows non-invasive and non-nephrotoxic evaluation of renal or non-renal arteries. MR angiography or venography is useful in the uremic patients who bear the highest risk of radiocontrast nephrotoxicity. During pregnancy, MRI may be used to analyze fetal uropathy.

Obstructive uropathy is a common etiology of renal failure in adults; in this setting, conventional IVU may be replaced by magnetic resonance urography to demon- strate ureter calculi or tumors. The CRISP cohort study has established the value of MRI in determining the respective volumes of kidney parenchyma and cysts in patients with polycystic kidney disease; interestingly, these parameters were significantly and inversely correlated with renal function parameters as assessed with creatinine value and MRI-based measures of renal blood flow and GFR [32]. In patients with familial medullary cystic disease, or with lithium-induced chronic nephropathy, MRI allows visualization of renal medullary cysts as small as $1-3 \mathrm{~mm}[33,34]$.

Diffusion-weighted imaging seems to have great potential in the renal consequences of renal artery stenosis, a condition in which the ADC is decreased. However, further observational studies are needed since this sign does not seem specific; it is also encountered in various causes of renal failure (interstitial fibrosis, tubular atrophy and glomerular disease) as well as in inflammatory conditions such as pyelonephritis [6].

USPIOs are cleared by macrophages in kidneys and in other soft tissues, and a decreased corresponding MRI signal is thus indicative of a local inflammatory process. Interestingly, an increased influx of macrophages in a rat model of kidney transplantation and in another of nephrotic syndrome was detected with MRI; the value of this technique in human inflammatory pathologies has clearly to be studied.

In summary, MRI has a tremendous potential, with the help of a new generation of contrast agents, to improve the diagnosis of acute as well as of chronic renal failure. Moreover, the new specific agents used in experimental studies help to better understand the mechanisms of renal insult, which will result in more appropriate and effective therapeutic options.

\section{References}

1 Dagher PC, Herget-Rosenthal S, Ruehm SG, Jo SK, Star RA, Agarwal R, Molitoris BA: Newly developed techniques to study and diagnose acute renal failure. J Am Soc Nephrol 2003;14:2188-2198.

- 2 Jeong JY, Kim SH, Lee HJ, Sim JS: Atypical low-signal-intensity renal parenchyma: causes and patterns. Radiographics 2002;22:833846.

-3 Chung JJ, Semelka RC, Martin DR: Acute renal failure: common occurrence of preservation of corticomedullary differentiation on MR images. Magn Res Imaging 2001;19:789_ 793.
4 Grenier N, Basseau F, Ries M, Tyndal B, Jones $\mathrm{R}$, Moonen C: Functional MRI of the kidney. Abdom Imaging 2003;28:164-175.

$\rightarrow 5$ Le Bihan D, Breton E, Lallemand D, Aubin ML, Vignaud J, Laval-Jeantet M: Separation of diffusion and perfusion in intravoxel incoherent motion MR imaging. Radiology 1988 ; 168:497-505.

6 Thoeny HC, De Keyzer F, Oyen RH, Peeters RR: Diffusion-weighted MR imaging of kidneys in healthy volunteers and patients with parenchymal diseases: initial experience. Radiology 2005;235:911-917.
7 Muller MF, Prasad PV, Bimmler D, et al: Functional imaging of the kidney by means of measurement of the diffusion coefficient. Radiology 1994;193:711-715

-8 Namimoto T, Yamashita Y, Mitsuzaki K: Measurement of the apparent diffusion coefficient in diffuse renal disease by diffusionweighted echo-planar MR imaging. J Magn Res Imaging 1999;9:832-837.

-9 Laissy JP, Menegazzo D, Dumont E, Piekarski JD, Karila-Cohen P, Chillon S, SchoumanClaeys E: Hemodynamic effect of iodinated high-viscosity contrast medium in the rat kidney: a diffusion-weighted MRI feasibility study. Invest Radiol 2000;35:647-652. 
10 Bennett HF, Li D: MR imaging of renal function. Magn Reson Imaging Clin N Am 1997;5: 107-126.

-11 Coulam CH, Lee JH, Weddling KL, Spielman DM, Pele NJ, Kee ST, Hill BB, Bouley DM, Derby GC, Myers BD, Sawyer-Glover AM, Sommer FG: Noninvasive measurement of extraction fraction and single-kidney glomerular filtration rate with MR imaging in swine with surgically created renal arterial stenoses. Radiology 2002;223:76-82.

12 Grenier N, Trillaud H, Combe C, Degreze P, Jeandot R, Gosse P, Douws C, Palussiere J: Diagnosis of renovascular hypertension: feasibility of captopril-sensitized dynamic MR imaging and comparison with captopril scintigraphy. Am J Roentgenol 1996;166:835-843.

-13 Lee VS, Rusinek H, Noz ME, Lee P, Raghavan M, Kramer EL: Dynamic three-dimensional MR renography for the measurement of single kidney function: initial experience. Radiology 2003;227:289-294.

$\checkmark 14$ Mandry D, Pedersen M, Odille F, Robert P, Corot C, Felblinger J, Grenier N, Claudon M: Renal functional contrast-enhanced magnetic resonance imaging: evaluation of a new rapidclearance blood pool agent (p792) in SpragueDawley rats. Invest Radiol 2005;40:295-305.

$\checkmark 15$ Dupas B, Buzelin MF, Karam G, Vasse N, Metlah K, Bach-Gansmo T: Contrast-enhanced MR imaging of experimental acute renal necrosis. Acta Radiol 2001;42:74-79.

- 16 Kobayashi H, Kawamoto S, Jo SK, Sato N, Saga T, Hiraga A, Konishi J, Hu S, Togashi K, Brechbiel MW, Star RA: Renal tubular damage detected by dynamic micro-MRI with a dendrimer-based magnetic resonance contrast agent. Kidney Int 2002;61:1980-1985.

17 Reith W, Forsting M, Vogler H, Heiland S, Sartor K: Early MR detection of experimentally induced cerebral ischemia using magnetic susceptibility contrast agents: comparison between gadopentetate dimeglumine and iron oxide particles. Am J Neuroradiol 1995; 16 : $53-60$.
18 Trillaud H, Degrèze $\mathrm{P}$, Combe C, Deminière $\mathrm{C}$, Palussière $\mathrm{J}$, Benderbous $\mathrm{S}$, Grenier $\mathrm{N}$ : USPIO-enhanced MR imaging of glycerol-induced acute renal failure in the rabbit. Magn Res Imaging 1995;13:233-240.

19 Franck H, Weissleder R, Brady TJ: Enhancement of MR angiography with iron oxide: preliminary studies in whole-blood phantom and in animals. Am J Roentgenol 1994;162:209213.

-20 Mergo PJ, Helmberger T, Nicolas AI, Ros PR: Ring enhancement in ultrasmall superparamagnetic iron oxide MR imaging: a potential new sign for characterization of liver tissues. Am J Roentgenol 1996;166:379-384.

21 Vexler VS, Berthezene Y, Clement O, Muhler A, Rosenau W, Moseley ME, Brasch RC: Detection of zonal renal ischemia with contrastenhanced MR imaging with a macromolecular blood pool contrast agent. J Magn Reson Imaging 1992;2:311-319.

22 Laissy JP, Benderbous S, Idee JM, Chillon S, Beaufils H, Schouman-Claeys E: MR assessment of iodinated contrast induced nephropathy in rats using ultrasmall particles of iron oxide. J Magn Reson Imaging 1997;7:164-170.

23 Laissy JP, Idee JM, Loshkajian A, Benderbous S, Chillon S, Beaufils H, Schouman-Claeys E: Reversibility of experimental acute renal failure in rats: assessment with USPIO-enhanced MR imaging. J Magn Reson Imaging 2000; 12: 278-288.

24 Lowenborg EK, Jaremko G, Berg UB: Glomerular function and morphology in puromycin aminonucleoside nephropathy in rats. Nephrol Dial Transplant 2000;15:1547-1555.

25 Hauger O, Delalande C, Trillaud H, Deminière C, Quesson B, Kahn H, Cambar J, Combe $C$, Grenier N: MR imaging of intrarenal macrophage infiltration in an experimental model of nephrotic syndrome. Magn Reson Med 1999;41:156-162.
26 Hauger O, Delalande C, Deminière C, Fouqueray B, Ohayon C, Garcia S, Trillaud $\mathrm{H}$, Combe C, Grenier N: Nephrotoxic nephritis and obstructive nephropathy: evaluation with MR imaging enhanced with ultrasmall superparamagnetic iron oxide. Preliminary findings in a rat model. Radiology 2000;217:819-826.

27 Strom TB, Carpenter CB: Immunobiology of kidney transplantation; in Brenner BM, Rector FC (eds): The Kidney, ed 4. Philadelphia, Saunders, 1991, pp 2336-2407.

- 28 Zhang Y, Dodd SJ, Hendrich KS, William M, Ho C: Magnetic resonance imaging detection of rat renal transplant rejection by monitoring macrophage infiltration. Kidney Int 2000;58: $1300-1310$

29 Hauger O, Delmas Y, Deminière C, Merville $\mathrm{P}$, Combe C, Grenier N: Late Sinerem-enhanced MR imaging of renal diseases: a pilot study. Eur Radiol 2004;14(suppl 2):202 (abstr B-349)

30 Yang D, Ye Q, Williams M, Sun Y, Hu TCC Williams DS, Moura JMF, Ho C: USPIO-enhanced dynamic MRI: evaluation of normal and transplanted rat kidneys. Magn Reson Med 2001;46:1152-1163.

31 Persson PB, Hansell P, Liss P: Pathophysiology of contrast medium-induced nephropathy. Kidney Int 2005;68:14-22.

32 King BF, Torres VE, Brummer BE, Chapman $\mathrm{AB}$, Bae KT, Glockner JF, Arya K, Felmlee JP, Graham JJ, Guay-Woodford LM, Bennett WM, Klahr S, Hirschman GH, Kimmel PL, Thompson PA, Miller JP; Consortium for Radiologic Imaging Studies of Polycystic Kidney Disease: Magnetic resonance measurements of renal blood flow as a marker of disease severity in autosomal-dominant polycystic kidney disease. Kidney Int 2003;64:2214-2221.

33 Farres MT, Ronco P, Saadoun D, Remy P, Vincent F, Khalil A, Le Blanche AF: Chronic lithium nephropathy: MR imaging for diagnosis. Radiology 2003;229:570-574.

- 34 Meier P, Farres MT, Mougenot B, Jacob L, Le Goas F, Antignac C, Ronco P: Imaging medullary cystic kidney disease with magnetic resonance. Am J Kidney Dis 2003;42:E5-E10. 